\title{
Research on Key Technologies of Wheeled Robot
}

$$
\text { Bin } \mathrm{HU}^{1 *} \text {, Jin-Peng XING }{ }^{2} \text { and Yong WANG }{ }^{1}
$$

\author{
${ }^{1}$ Shanghai Fire Research Institute of Ministry of Public Security, Shanghai 200438, China \\ ${ }^{2}$ Department of Mechanical and Electronic Engineering, Dongchang College of Liaocheng \\ University, Shandong Liaocheng 252000, China
}

Keywords: Wheeled robot, Single-wheel, Two-wheeled, Rolling robot.

\begin{abstract}
As the main carrier of the robot, the wheeled robot has been studied for many years. Wheeled robots are driven by wheels to drive the robot to move and work. The key technologies of single-wheel rolling robot, two-wheeled mobile robot, three-wheeled four-wheeled robot and sixwheeled robot are reviewed in this paper. Finally, the future development trend of wheeled robots is prospected.
\end{abstract}

\section{Introduction}

Wheeled robots are driven by wheels to drive the robot to move and work. Although its motion is closely related to road conditions, it is widely used because of its light weight, large bearing capacity, simple mechanism, relatively easy drive and control, high running speed and high working efficiency[1-5].

At the end of twentieth Century, the design of the driving mechanism has been a difficult problem in the development of robots. Since then, a large number of design research has been done home and board, in a short period of less than 20 years, and has solved the problem of wheeled robot transmission mechanism [6-8]. Wheeled robots have wide applicability in teaching, scientific research, field work and civil transportation. They are of great value in anti-terrorism and other cutting-edge fields.

Due to the important role of wheeled mechanism in space detection robot system, many foreign institutions are studying robot wheel mechanism. There are relatively well-known research institutions: Carnegie Mellon University, the Jet Propulsion Laboratory, Finland Helsinki University of Technology and the European Space Agency. Overall, in the domestic field of wheeled robot has made a lot of achievements, the design of wheeled robots are able to maintain the normal movement in the unpredictable environment, able to adapt to the unknown environment is complex and changeable, can deal with various emergency situations and has strong ability of avoiding the obstacle to the successful completion of the task.

\section{Wheeled Robot Classification}

As the number of wheels, directly related to the robot design technology and the difficulty, and its function. So the classification of wheeled robots are generally based on the number of wheels to be classified. According to the robots have emerged, can be divided into the following categories: single-wheel rolling robot (such as spherical robot), two mobile robots (such as bicycle robots), three or four robot (such as smart cars), six-wheeled robot and composite wheel robot. In general, three-wheeled robot is simple and practical, four-wheel robot stability, carrying capacity, and in contrast, six-wheel robot is more superior than the four-wheeled robot.

\section{Single-Wheel Rolling Robot}

\section{Background}

Single-wheel rolling robot is a new concept of wheeled robots. From the exterior point of view it is only a wheel, its movement is rolling along the ground forward, and later developed a ball-type robot is also a single-wheel rolling robot[9-12].

The research work of single-wheel rolling robot mainly includes: 
(1) The dynamic model of the single-wheel rolling robot and the coupling and parameterization of propulsion force and manipulator;

(2) motion information acquisition method based on position sensor;

(3) dynamic stability and static instability of the control program.

The research of single-wheel rolling robot has wide application prospect:the use of its amphibious characteristics, it will be introduced to the beach and swamps and other environments, for transportation, rescue and mineral exploration;in the field of spaceflight, based on the principle of single-wheel rolling robot, a lunar rover can be developed which is free from the influence of terrain and motion.

\section{Design of Main Structure}

The main body of the robot as a single-wheel mobile robot core, the need to complete the functions are: (1) the acquisition of the main body of the attitude data; (2) drive circular sphere movement; (3) for the entire system power supply. So the robot body needs to contain the robot control panel, the robot drive device and the system power supply three parts. At the same time, in order to be more conducive to the robot's balance and stability, the main body of the various devices are installed on the main body as the central symmetry point for symmetric installation. The schematic diagram of the robot main body structure is shown in the figure 1 .

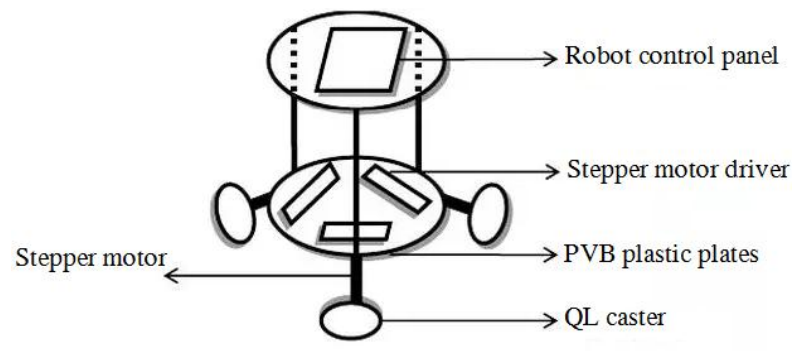

Figure 1 . The schematic diagram of the robot main body structure.

\section{Two-Wheeled Mobile Robot}

The two-wheeled robot consists of a bicycle robot and two wheeled mobile robots arranged symmetrically.- 10-point Times, Times Roman or Times New Roman. The mechanical design of wheeled robots belongs to mechanical field. In the process of design, not only the influence of own weight but also the influence of working environment is taken into account, and the data collection and analysis can not be disturbed[13].

The overall structure of the double-wheeled robot is shown in the figure 2 . The structure of the two-wheeled robot is connected by a hollow arc tube into a "I" shape, and a stepping motor is built in each wheel center to control the reversal of the wheel. In the two sides of the inside of a hanging basket and hollow tube fixed as a whole, hanging basket with battery blocks, both as a power source, can also be used as a weight block. The hanging basket and the hollow pipe are integrally connected by screws to facilitate disassembly. In the middle of the hollow tube has a camera, used to observe the feedback site. By remote control of motor steering, can easily achieve the robot forward, backward, and in situ steering. The bottom of the basket is equipped with a battery block, which can play the role of weight block, to ensure that the wheeled robot in the flat road or slope can be maintained at rest. 


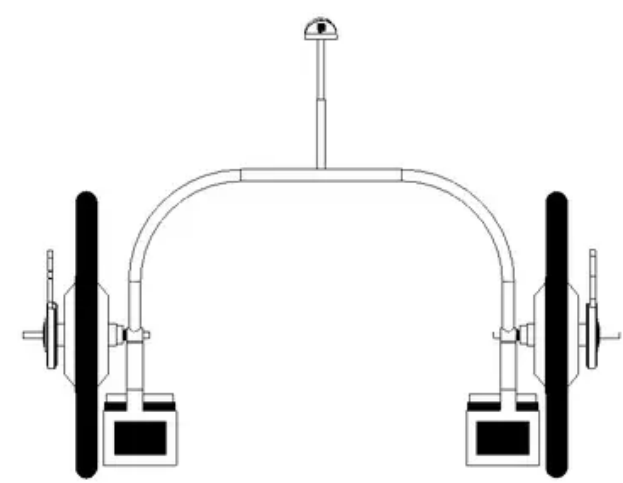

Figure 2. The overall structure of the double-wheeled robot.

\section{Three-Wheel and Four-Wheel Robots}

The most common mechanism in wheeled robots is the three- and four-wheeled robots. This robot is the most suitable choice when walking on flat terrain, and has provided mature technology for its development in other fields (such as automotive)[14-16].

The three-wheeled mobile robot is similar to four-wheeled mobile robot. It is divided into steering wheel and rear wheel driven by front wheel, front wheel driven by front wheel, front wheel driven by caster, front wheel driven by caster, The latter two wheels are driven by a motor, in order to achieve differential steering these three ways. So far, many researches have been done on threewheeled and four-wheeled mobile robots, mainly related to robot mechanism, architecture, motion planning, navigation and positioning, tracking control, feedback stabilization of motion control, interactive technology, multi-sensor system and information Integration, intelligent technology and other key technologies. At the same time, this kind of robot research also provides the foundation for the development of multi-wheel and compound robots. And will have a profound impact on the development of modern automobile industry. The three wheeled mobile robot is stable, and is simple and practical, so far, there are a lot of research on the three wheeled mobile robot, mainly related to the robot mechanism, system structure, motion planning, navigation and positioning, tracking control, feedback control, motion control, interactive technology, multi sensor system and information fusion, the key technology of intelligent technology.

Three wheeled mobile robot can be divided into four types according to the different combinations of wheel configuration and function, which can be shown as figure 3 .

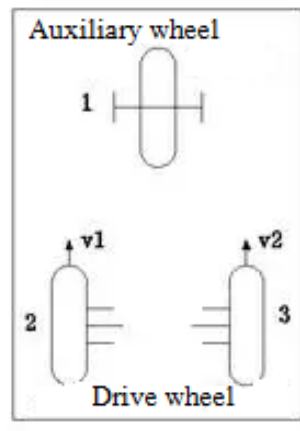

(a)

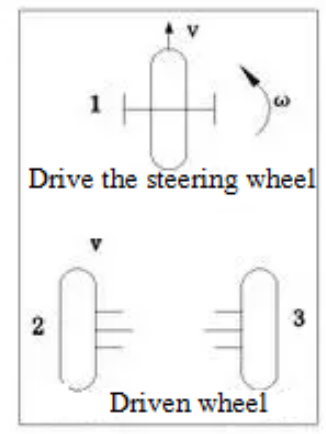

(b)

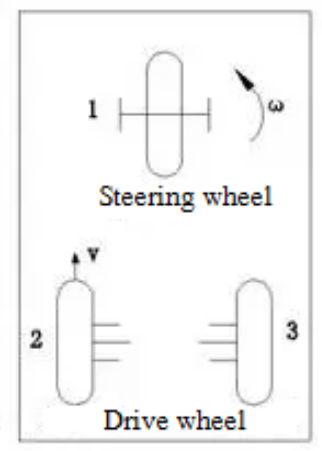

(c)

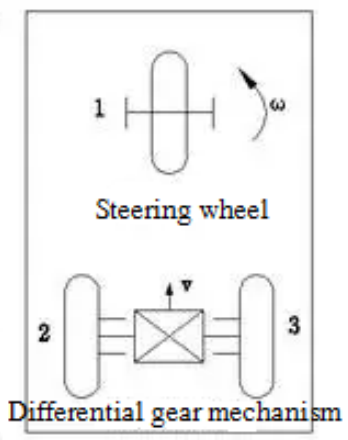

(d)

Figure 3. Four configurations for three-wheeled mobile robot.

\section{Six-Wheel Robots}

Compared with four-wheel machines, six-wheel robots have higher mobility and reliability, and better off-road capability, but the structure is relatively complicated. The representative is the USdeveloped Mars exploration vehicle, which uses six-wheel rocker suspension mechanism, which adopts symmetrical structure. The one-side rocker arm mainly includes the main rocker arm, the 
secondary rocker arm, the front and rear two driving wheels and the middle follow-up wheel. Compared with the four-wheel structure, due to the introduction of the secondary rocker and driven wheel, when obstacles, through the deputy rocker rotation, and by means of the follower wheel to adjust the gravity on each wheel component, can improve the car Body stability and off-road capability[17-20].

Robot is the implementation of mobile robot and complete the task of carrying platform, as the core part of walking robot system, its design quality is an important indicator of the performance of the robot system. The design of the walking mechanism is mainly composed of the suspension system, the chassis system, the transmission system bearing wheel mechanism and the walking driving mechanism. In the design process of robot walking mechanism, we must first comply with the following principles: (1) The size of the body to meet the design requirements; (2) Parts for easy processing, measurement; (3) The design of the body parts to meet the strength and stiffness requirements; (4) Designed for easy installation, commissioning and repair; (5) As the robot's mobile platform, for the sensor, functional components and other reserved space and location;(6) To ensure that all the assembly parts and assembly components are easy to be assembled and disassembled, and the guarantee mechanism can meet the design requirements and not interfere with each other.

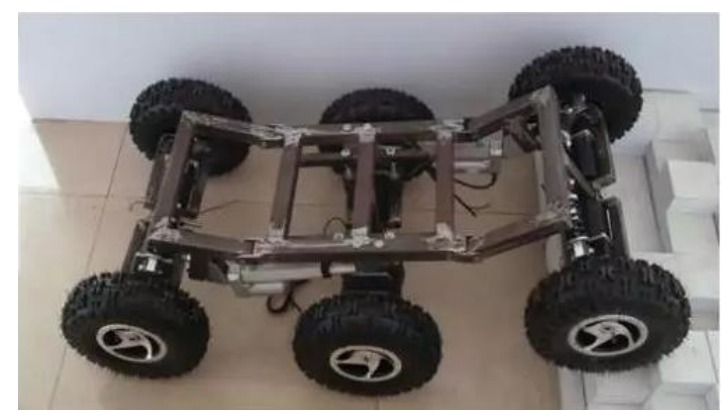

Figure 4. Six-wheel robot physical map.

\section{Summary and Prospect}

As robotic applications continue to expand, the operation of robots becomes more complex. Conventional wheeled robot configurations cannot meet many special operating requirements. In order to meet the specific operational requirements, the need to design a new type of robot structure, in order to achieve accurate and rapid expected movement. Wheeled robot technology has the extensibility and some versatility, can be applied to other areas. Such as military mine clearance, robotic robots, exploration robots, environmental robots and rescue robots. Wheeled robot is a combination of robotic technology and intelligent control technology. Therefore, the research and realization of wheeled robots are of great significance to promote the development of robotics, intelligent robot technology and intelligent control technology.

Nowadays, wheeled robots designed to move in unstructured environment have higher requirements. The design of wheeled robots should be small in size, light in weight, compact in structure and strong in adaptability to terrain. In general, the development trend of wheeled robots is:

(1)Wheeled robot structure is no longer a simple wheel structure, replaced by a strong nonstructural environment to adapt to the ability of wheel, leg, crawler-type composite structure and can be realized wheel structure.

(2)Due to the limitations of robot technology, size, quality and cost, miniature and small-sized wheeled robots are the mainstream of the current development.

\section{Acknowledgements}

This work was supported by Shanghai Science and Technology Commission [grant number 14dz1206802]. 


\section{References}

1. C. L. Hwang,C. Y. Shih,IEEE T IND ELECTRON 597 (2009)

2. A. V. Pesterev,AUTOMAT REM CONTR 709 (2009)

3. A. Abbaspoura, K. Alipourb,H. Z. Jafaria, S. Ali,A. Moosavian,Comptes Rendus Mécanique 343 5-6 (2015)

4. Y.j. Kim, B. K. Kim,ROBOT AUTON SYST 64 (2015)

5. A. V. KarapetyanM. A. Salmina,Moscow Uni Mech Bull 636 (2008)

6. Y. Kim,S. H. Kim,Y. K. Kwak,J. Int. Rob. Sys 441 (2005)

7. S. Kim, S. Kwon,Int. J. Con. Auto. Sys 134 (2015)

8. F. Grasser, A. D. Arrigo, S. Colombi, and A. C. Rufer, IEEE Trans. on Industrial Electronics 491 (2002)

9. T. Takei, R. Imamura, and S. Yuta, IEEE Trans. on Industrial Electronics,56 10 (2009)

10. G. C. Anousaki,K. J. Kyriakopoulos,IEEE Robot. Autom. Mag 14 1(2007)

11. H. Durrant-Whyte,T. Bailey,IEEE Robot. Autom. Mag 132 (2006)

12. R. O. Ambrose, R. T. Savely, S. M Goza, P. Strawser, M. A. Diftler, I. Spain, and N. Radford, Proc. of IEEE ICRA (2004)

13. S. Jung, T. C. Hsia, and R. G. Bonitz, IEEE Trans. on Control Systems Technology 122 (2004) 14. T. C. Lee, K. T. Song, C. H. Lee, and C. C.Teng, IEEE Trans. on Control Systems Technology $92(2001)$

15. F. Abdessemed, K. Benmahammed,E. Monacelli, Auton. Syst 475 (2004)

16. A. K. Khalaji,S. A. A. Moosavian,J MECH SCI TECHNOL 301 (2016)

17. M. H. KorayemR. Abbasi EsfedenS. R. Nekoo,J MECH SCI TECHNOL 294 (2015)

18. G. Michalos, K. Kaltsoukalas, P. Aivaliotics, P. Sipsas, A. Sardelis and G. Chryssolouris, CIRP Ann-Manu Tech 631 (2014)

19. F. Abdessemed, K. Benmahammed and E. Monacelli,ROBOT AUTON SYST 471 (2004)

20. M. H. Korayem, M. Irani and S. R. Nekoo,AMAE Int. J. Manu. Mate. Sci 21 (2012) 